\title{
Eine neue Methode der quantitativen Spektralanalyse.
}

\author{
Von \\ G. und H. KRüss. ${ }^{1}$ \\ Mit einer Figur im Text.
}

\section{Einleitung.}

Die von VIERonds zuerst in gröfserem Mafse in die Analyse eingeführte spektrophotometrische Methode wird bekanntlich mit Erfolg angewendet bei einer Reihe von speziellen analytischen Bestimmungen, bei physiologischen Arbeiten, sowie in manchen technischen Untersuchungen.

Das Gebiet ihrer Anwendung ist naturgemäls ein weites, weil eine Unzahl von Verbindungen an sich gefärbt sind oder mit Leichtigkeit in gefürbte ïbergeführt werden können.

VIERORD' selbst hat in seinen beideu grundlegenden Arbeiten „Die Anwendung des Spektralapparates zur Photometrie der Absorptionsspektren und zur quantitativen chemischen Analyse" (Tübingen 1873) und ,Die quantitative Spektralanalyse in ihrer Anwendung auf Physiologie, Physik, Chemie und Technologie" (Tübingen 1876) cine grofse Anzahl von Beispielen gegeben, in welchen die quantitative Spektralanalyse nutzbringend angewendet wird, HüFNER ist

I In den nachfolgenden Mitteilungen gebe ich das Resultat von Überlegungen, welche mein verstorbener Bruder mit mir im Herbst 1894 gepflogen hat. Es ist die letzte Frucht des schönen, seit Jahren immer imniger gt:wordenen Verhältuisses zwischen uns, welches auf gegenseitigem Verständuis unserer wissenschaftlichen Bestrebungen beruhte. Der Anstofs dazu stammt von meinem Bruder her, weleher durchdrungen war von der Wichtigkeit der quantitativen Spektralanalyse, welcher aber durch die Erfahrung mit einer Reile von Schïlern, die er in dieselbe einführte, zu der Überzengung grekummen war, dals diese Methode einfacher und durchsichtiger gestaltet werden müsse, um ihr gröfseren Eingang in die Kreise der Chemiker zu verschaffen. Er wurde darin bestärkt durch Zweifel an der Richtigkoit der benutzten Formeln, welche H. SAlkowski-Münster ihm gegenüber brieflich im letzten Sommer aussprach. Die vorliegende Arbeit ist von meinem Bruder so weit gefördert worden, dal's zwischen uns bereits das Schema für die Ausarbeitung festgestellt war, so dafs nur noch letztere ohne seine Beihilfe von mir gemacht worden ist. H. Krüss. 
ihm auf physiologischem Gebiete darin nachgefolgt und in unserem Buche „Kolorimetrie und quantitative Spektralanalyse" (Hamburg und Leipzig 1891) ist eine möglichst vollständige Darstellung des damaligen Standes dieser Untersuchungsmethode gegeben worden. Trotzdem hat die Methode der quantitativen Spektralanalyse nicht die weitverbreitete Anwendung erfahren, wie sie es verdiente und wie andere Zweige der analytischen Chemie, z. B. die Gasanalyse, sie aufzuweisen haben.

Wir baben uns den Grund dieser eigentümlichen Erscheinung klar zu machen gesucht und sind der Meinung, dals derselbe darin liegt, dals sowohl die theoretischen Grundlagen dieser Methode wie die instrumentellen Anordnungen, welche ihre Anwendung ermöglichen, nicht in dem Grade durchsichtig und einfach zu erfassen sind, wie es nötig ist, um einer Arbeitsmethode mit Leichtigkeit Eingang in die tägliche Praxis zu verschaffen.

\section{Bisherige Grundlage und Anordnung der quantitativen Spektral- analyse.}

Es kaun hier nicht darauf verzichtet werden, kurz diejenigen Entwickelungen anzuführen, welche bisher als Grundlage der quantitativen Spektralanalyse gedient haben.

Es ist vor allem das LamberTsche Gesetz, auf welches alle Überlegungen aufgebant werden müssen. Nach demselben wird die ursprüngliche Helligkeit $J$ nach dem Durchlaufen einer Schicht von der Dicke $m$ auf den Betrag

$$
J^{\prime}=\frac{J}{n^{m_{1}}}
$$

gebracht, wenn der Schwächungsfaktor für die Einheit der Dicke $\frac{1}{n}$ ist. Die Richtigkeit dieser Beziehung ist durch vielfache Versuche nachgewiesen worden. ${ }^{1}$ Sie gilt in voller Strenge allerdings nur bei der Anwendung homogenen Lichtes, da bei gemischtem Lichte an die Stelle des obigen einfachen Ausdruckes die Summe

$$
\frac{J_{1}}{n_{1}{ }^{m}}+\frac{J_{9}}{n_{2}{ }^{m}}+\frac{J_{3}}{n_{3}{ }^{m}}+\ldots
$$

treten würde, in welcher sich die Indices auf die verschiedenen Komponenten des gemischten Lichtes bezieher.

S. $69 \mathrm{ff}$.

${ }^{1} \mathrm{Vgl}$. G. und H. KRƯss, Kolorimetrie und quantitative Spektralanalyse, 
Setzt man die ursprüngliche Lichtstärke gleich der Einheit, so ist

$$
J^{\prime}=\frac{1}{n^{n i}}
$$

also

$$
\log n=-\frac{\log J}{m} \text {. }
$$

Zur Vereinfachung der Berechnung der Konzentration einer Lösung aus der durch dieselbe hervorgerufenen Lichtabsorption führten BUNSEN und Roscos ${ }^{1}$ bekanntlich den Extinktionskoëffizienten $e$ ein, welchen sie definierten als den reciproken Wert derjenigen Schichtendicke, welche eine Substanz haben mufs, um das durch dieselbe fallende Licht bis auf $1 / 10$ der Intensität durch Absorption abschwächen zu können.

Setzt man also entsprechend dieser Definition in Gleichung (2)

$$
m=\frac{1}{e} \text { und } J^{\prime}=\frac{1}{10},
$$

so wird

$$
\log n=e
$$

also

$$
e=-\frac{\log J^{\prime}}{m}
$$

Sodann ist man zur Vereinfachung der Berechnung übereingekommen, stets mit der Schichtendicke $m=1 \mathrm{zu}$ arbeiten, und erhält infolgedessen

$$
e=-\log J^{\prime}
$$

d. h. der Extinktionskoëffizient ist gleich dem negativen Lagarithmus der übrigbleibenden Helligkeit. Die Grölsen dieser negativen Logarithmen sind in Tabellen niedergelegt, welche den in der Einleitung genannten ausführlichen Schriften über die quantitative Spektralanalyse beigegeben sind. Es gestaltet sich also das Endresultat recht einfach, indem nur die durch eine Lösung von der Dicke 1 hindurchgegangene Lichtmenge bestimmt und aus der vorhandenen Tabelle der zugehörige Extinktionskoëffizient entnommen zu werden braucht.

So wenig nun die Richtigkeit der vorstehenden Entwickelung angezweifelt werden kann, so ist es doch zweifellos verwirrend, namentlich für den Anfänger, dals man zur Gewinnung des Begriffs des Extinktionskoëffizienten die Herbeiführung einer Lichtschwächung auf $1 / 10$ der ursprünglichen Lichtstärke voraussetzt, also eigentlich die Dicke der durchstrahlten Schicht variabel machen mülste, anstatt dessen aber die Dicke konstant gleich der Einheit macht und

1 Pogg. Ann. (1857) 101, 238.

z. anorg. Chem. $\mathrm{X}$. 
den nunmehr natürlich von dem Werte $1 / 10$ verschiedenen Betrag der übrigbleibenden Lichtstärke milst. Es geht dabei leicht das klare Bild des eigentlichen physikalischen Vorganges verloren, es bleibt der Extinktionskoëffizient ein schwieriger, der Anschauung nur schwer zugänglicher Begriff.

Will man aus dem gefundenen Extinktionskoëffizienten auf die Konzentration einer Lösung schlielsen, so mufs zwischen beiden nach einer Beziehung gesucht werden. Bonsex und Roscos haben bereits experimentell nachgewiesen, dals die Vermehrung der Konzentration denselben Einflufs auf die Lichtabsorption ausübt, wie die Vergrölserung der Schichtendicke. Man kann sich eine Lösung von der Konzentration 0 denken als bestehend aus $e$ einzelnen Schichten von der Konzentration 1. Dals in der That sich die Sache so verhält, haben die Versuche von Vierondr und die vielen Bestimmungen von G. Krüss nachgewiesen, wobei natürlich immer vorausgesetzt werden mufs, dafs keine Dissoziation stattfindet. Die Dicke, welche eine Lösung haben mufs, um die übrigbleibende Lichtstärke auf $1 / 10$ ihres ursprünglichen Wertes herunterzubringen, muls also um so kleiner sein, je stärker die Konzentration $c$ der Lösung ist, der Extinktionskoëffizient, d. i. der reciproke Wert dieser Dicke, muls also in demselben Verhältnis wachsen wie die Konzentration, d. h. das Verhältnis der Konzentration zum Extinktionskoëffizienten $e$ muls ein konstantes sein. VIERorde bezeichnete dieses Verhältnis als Absorptionsver$h a ̈ l t n i s(A)$, welches sich berechnet aus der Gleichung

$$
\frac{c}{e}=A \text {, }
$$

so dals, wenn die Konstante $A$ ein für alle Mal für den betreffenden in Lösung befindlichen Körper bestimmt und $e$ auf irgend eine spektrophotometrische Weise für eine bestimmte Lösung dieses Körpers gefunden ist, die Konzentration derselben aus der einfachen Beziehung

$$
e=A \cdot e
$$

hervorgeht.

Auch in der Ableitung des Absorptionsverhältnisses wird wiederum Bezug genommen auf die Herbeiführung der Lichtschwächung auf $1 / 10$ des ursprünglichen Wertes, welche bei. der Ausführung der Versuche thatsächlich nicht hergestellt wird, da man immer mit der konstanten Dicke 1 arbeitet. Wir betonen, dafs natürlich in den gegebenen Ableitungen keinerlei Denkfehler vorhanden ist, wieder- 
holen aber, dals sie eine Denkschwierigkeit darbieten, wegen deren mancher Praktiker schon bei dem Versuch, sich in die Grundlagen der quantitativen Spektralanalyse hineinzuarbeiten, gescheitert und so nicht zur praktischen Anwendung derselben gekommen sein mag.

Was nun die experimentelle Anordnung der Untersuchungen auf dem Wege der quantitativen Spektralanalyse anbelangt, so wird mit Hilfe irgend eines Spektralphotometers der Extinktionskoëffizient einer vor den Spalt gestellten Lösung in der Schichtendicke 1 bestimmt. Die Ausführung dieser Bestimmung geschieht in der Weise, dal's die eine Hälfte des den Spalt eines Spektralapparates von irgend einer Lichtquelle treffenden Strahlenbündels ungeschwächt in den Apparat eindringt, während die andere Hälfte durch die Lösung tritt und durch diese geschwächt wird. Sodann werden in melsbarer Weise die im Okular des Apparates sichtbaren beiden verschieden hellen Hälften des Spektrums auf die gleiche Helligkeit gebracht, indem entweder die Helligkeit des nicht durch die Lösung gegangenen Strahlenbündels verringert oder die des anderen vergröfsert wird und auf diese Weise die durch die Absorption in der Lösung übrig gebliebene Lichtstärke gemessen.

Zur Schwächung der nicht durch die Lösung gegangenen Strahlen dienen in den Polarisationsspektrophotometern polarisierende Mittel. Unser allgemeines Urteil über die Eigenschaften dieser Apparate, namentlich über ihre Helligkeit ist aus unseren früheren Veröffentlichungen bekannt. Hier sei nur als Hindernis in der praktischen Anwendung hervorgehoben, dals gerade der Chemiker häufig nicht in der Lage ist, die Wirkungsweise eines solchen Apparates zu übersehen. Wohl wollen wir mit dieser Bemerkung nicht den physikalischen Bildungsgrad der Chemiker überhaupt herabsetzen; wir wissen ja sehr wohl, dals gerade die Handhabung von Polarisationsapparaten zum täglichen Betriebe manchen chemischen Laboratoriums gehört. Aber es lälst sich nicht bezweifeln, dafs es bei Einführung einer neuen Untersuchungsmethode in die tägliche Praxis des Chemikers vor allem darauf ankommt, die dazu nötigen Werkzeuge so zu gestalten, dafs er ihre Wirkungsweise von vornherein übersehen kann, dafs dieselbe in Zusammenhang steht mit der sonst bei ihm üblichen Art des Arbeitens. Nur dann wird man darauf rechnen können, dafs er einer solchen Methode von vornherein Vertrauen entgegenbringt und sich mit Bereitwilligkeit ihr zuwendet.

Bei der von Vierordt empfohlenen Methode des Doppelspaltes, 
welcher wir aus praktischen Gründen den Vorzug vor den Polarisationsspektrophotometern geben, wird die Lichtschwächung der nicht durch die Lösung gegangenen Strahlen durch eine entsprechende Verengerung derjenigen Hälfte des Spaltes, auf welche sie fallen, bewirkt. Hier ist der Vorgang der Lichtschwächung sehr klar ersichtlich und durch das Ablesen der Spaltbreite erhält man direkt die Gröfse der Lichtschwächung. Nur. ist der Doppelspalt ein sehr empfindlicher Melsapparat und es muls von dem Chemiker, welcher ihn benutzt, verlangt werden, dafs er vor Beginn seiner Untersuchungen stets prüfe, ob sein Doppelspalt noch in vollkommener Ordnung sei, d. h. ob die beiden Hälften desselben bei Einstellung auf Null rollkommen geschlossen sind und ob bei gleich weiter Öffnung beider Hälften die beiden Hälften des Spektrums gleiche Helligkeit besitzen. So einfach und selbstverständlich diese Forderungen sind, so mögen sie dennoch manchmal von dem ausübenden Chemiker vernachlässigt und so ein zuverlässiges Ergebnis seiner spektrophotometrischen Bestimmung in Frage gestellt werden.

Die bisher angeführten Gründe haben uns dazu veranlalst, zunächst eine einfachere Entwickelung der Grundlagen der quantitativen Spektralanalyse zu suchen. Dabei wurden wir dann weiter auf eine experimentelle Anordnung geführt, welche dem von uns eingeschlagenen Gedankengange entspricht und gleichzeitig, nach unserer Meinung, den ausübenden Chemiker mehr anmuthen wird als die bisherigen Anordnungen.

\section{Neue Methode der quantitativen Spektralanalyse.}

Es ist von vornherein klar, dafs wir bei unserer Entwickelung von denselben Grundsätzen ausgehen müssen wie bisher und dals es sich nur um eine andere Anordnung dabei handeln kann. Diesé Grundsätze sind einerseits das LAMBERTsche Gesetz und andererseits die Annahme, dals die Konzentration auf das eine Lösung durchdringende Licht in derselben Weise wirkt wie die Dicke derselben. Wir haben diesen Zusammenhang bereits in Vorstehendem ausführlich dargelegt und wiederholen nur, dafs die Gröfse der Lichtschwächung genau die gleiche ist bei einer Lösung von der Konzentration $o$ in der Dicke $m$, oder bei einer solchen von der Konzentration $m$ und der Dicke $e$.

Es sei also wieder wie früher unter $J$ die Intensität des auffallenden Lichtes, unter $J^{\prime}$ diejenige verstanden, welche nach Durchstrahlung der Schicht einer'Lösung von der Dicke $m$ und der Kon- 
zentration $c$ noch vorhanden ist. Bei der Durchstrahlung einer Lösung desselben Körpers in der Dicke 1 und der Konzentration 1 möge das Licht auf $\frac{1}{n}$ seiner ursprünglichen Intensität heruntergebracht werden.

Dann können wir sofort setzen

$$
J^{\prime}=\frac{J}{n^{m \cdot c}} .
$$

Nimmt man nun an $J^{\prime}=\frac{J}{x}$, so erhält man

$$
\frac{J}{x}=\frac{J}{n^{m \cdot c}}
$$

oder

$$
x=n^{m \cdot c}
$$

$$
\begin{aligned}
\log x & =m \cdot e \cdot \log n \\
e & =\frac{\log x}{m \cdot \log n}
\end{aligned}
$$

Diese an sich schon einfache Beziehung wird noch leichter zu handhaben, wenn $\operatorname{man} x=10$ setzt, das heifst wenn man eine solche Versuchsanordnung voraussetzt, dafs die Intensität des auf die Lösung fallenden Lichtes durch Absorption in der Lösung gerade auf $1 / 10$ ihres ursprünglichen Wertes gebracht wird. Dann wird $\log x=1$ und

$$
c=\frac{1}{m \log n} .
$$

Hierin ist $\log n$ für ein und denselben Körper eine Konstante, denn es wurde $\frac{1}{n}$ als Schwächungskoëffizient einer Lösung von der Dicke 1 und der Konzentration 1 definiert. Wir bezeichnen infolgedessen $n$ als das "spezifische Lichtabsorptionsvermögen"6 der Substanz und können die Grölse $\frac{1}{\log n}$ als Konstante $k$ bezeichnen. Dann ist

$$
c=\frac{k}{m},
$$

d. h. es kann die Konzentration einer Lösung durch Messsung ihrer Schichtendicke bestimmt werden. $\mathrm{Zu}$ dem $\mathrm{Z}$ wecke mufs $k$ bekannt sein. Es wird leicht ermittelt durch eine Messung an einer Lösung von bekannter Konzentration $c$, daraus findet sich

$$
k=m . c \text {. }
$$

Die von uns zunächst als $k$ bezeichnete Grölse ist nun keine neue Konstante, sondern sie ist genau dasselbe, wie das von VIER- 
ORDT mit $A$ bezeichnete Absorptionsverhältnis. Das geht aus folgender Uberlegung hervor.

Es war die Gleichung (8)

also

$$
c \leq \frac{\log x}{m} \cdot \frac{x}{\log n}
$$

$$
\frac{1}{\log n}=k=\frac{c}{m \log x} .
$$

Hält man sich nun gewärtig, dal's bei Definition des Vierondrschen Absorptionsverhältnisses immer eine Dicke $m=1$ vorausgesetzt war, so würde in dem Ausdruck rechter Hand in der Gleichung (11) nur $\frac{c}{\log x}$ nachbleiben. Das ist aber dieselbe Grölse, welche in Gleichung (5) mit $A$ bezeichnet worden ist, nämlich das Verbältnis der Konzentration zum negativen Logarithmus der übrigbleibenden Lichtstärke; $\log x$ ist in der That der negative Logarithmus der von uns mit $-\frac{1}{x}$ bezeichneten übrigbleibenden Lichtstärke. Deshalb können wir $k$ durch das bekannte und durch Versuche über viele Körper bereits ermittelte $A$ ersetzen ${ }^{2}$ und schreiben

$$
c=\frac{A}{m},
$$

ebenso wie $A$ gefunden wird aus der Gleichung

$$
A=c . m \text {. }
$$

Wenn also die Konstante $A$ bereits bekannt oder durch Messung nach dem im folgenden zu beschreibenden Verfahren mit Hilfe ron Gleichung (10a) aus einer Lösung von bekannter Konzentration und gemessener Dicke bestimmt ist, so kann man durch alleinige Bestimmung derjenigen Schichtendicke $m$, durch welche eine Lichtschwächung auf $1 / 10$ ihres ursprünglichen Wertes bewirkt wird, auf einfache Weise aus Gleichung (9a) die Konzentration $c$ der untersuchten Lösung bestimmen.

Für die experimentelle Anordnung eines dahingehenden Ver. suches handelt es sich also um zweierlei: man mul's die Anordnung so treffen, dafs bei Helligkeitsgleichheit eine Schwächung der ur-

${ }^{1}$ Es ist vornehmlich die Rücksicht darauf, das grolse Material, welches bereits in Bezug auf die Kenntnis der Vierondschen Grölse 1 vorliegt, ohne weiteres benützen zu könen, weshalb wir in Gleichung (8) $x=10$ angenommen haben. Um die von uns gewiinschte einfache Beziehung zu erhalten, hätte es offenbar geniigt, wenn man die Grölse $r$ überhaupt nur als eine in allen Fällen konstante annahm und für deren Wert irgend eine /ahl setzte, welche ans irgend einem praktischen Grumrle den Vorzug vor allen anderen verdiente. 
sprünglichen Lichtmenge auf $1 / 10$ stattfindet und man muls die Dicke der durchstrahlten Schicht in weitem Mals variieren können, um Helligkeitsgleichheit herzustellen, sowie endlich diese Dicke messen.

Der ersten Bedingung wird dadurch entsprochen, dafs von vornherein die eine Hälfte des im Gesichtsfelde des Beobachtungsfernrohres des Spektrophotometers erscheinenden Spektrums genau 10 mal so hell erscheint als die andere. Läfst man dann das Licht, welches die ursprünglich hellere Hälfte belenchtet, durch die Schicht der Lösung gehen und stellt ihre Dicke $m$ so ein, dal's nunmehr Helligkeitsgleichheit in beiden Hälften des Spektrums stattfindet, so hat die Lösung thatsächlich die Helligkeit auf $1 / 10$ heruntergehracht und ihre Konzentration ergiebt sich aus der einfachen Gleichung $e=\frac{A}{m}$.

Um von Anfang an das erforderliche Helligkeitsverhältnis von $1: 10$ in den beiden Spektralhälften herzustellen, kann man an einem der üblichen Polarisationsspektrophotometer den polarisierenden Mitteln die entsprechende Einstellung geben, und ebenso bei einem Spektrophotometer mit VIKRORDTschem Doppelspalt (Universalspektralapparat ${ }^{1}$ die eine Spalthälfte auf eine 10 mal so grofse Breite einstellen wie die andere.

Dann würde es also nur noch darauf ankommen, eine Flüssigkeitszelle mit variabler Dicke der Schicht zu benutzen. Denkt man sich dieselbe horizontal angeordnet, so kommt man auf den Gedanken eines keilförmigen Gefälses, welches mit der betreffenden Lösung gefüllt und vor der einen Hälfte des Spaltes in horizontaler Richtung bewegt wird. Natürlich mülste vor der anderen Hälfte in derselben Weise ein ebenso gestalteter, aber mit der Lösungsflüssigkeit gefüllter Keil bewegt werden.

Denkt man an eine derartige Anord̀nung, dals die Lösung und die Lösungsflüssigkeit wie bei einem gewöhnlichen Kolorimeter sich in vertikal aufgestellten Mensuren befinden, in denen die Höhe der Schicht auf irgend eine Weise verändert werden kann, so könnte man vor jede Hälfte des Spaltes ein Vergleichsprisma setzen und die beiden Mensuren seitswärts aufstellen, oder wie in dem LummezBrodhurschen Spektrophotometer ${ }^{2}$ zwei Kollimatoren mit je einem Spalt benützen, oder auch nach der von $P$. Schottuländer ${ }^{3}$ vor-

\footnotetext{
1 Ber. deutsch. chem. Ges. (1886) 19, 2739.

2 Zeitschr. Instrum. Kunde (1892) 12, 132.

${ }^{3}$ Zeitscher. Instrum. Kunde (1889) 9, 98.
} 
geschlagenen Art den Kollimator durch eine horizontal in der optischen Achse liegende Scheidewand in zwei halbe Kollimatoren teilen. Alle diese Anordnungen sind möglich und anwendbar, wemn man nur dafür sorgt, dafs von vornherein auf beide Flüssigkeiten die gleiche Lichtmenge auffällt, und bei ihnen allen genielst man den Vorteil des entwickelten einfachen Zusammenhanges zwischen Schichtendicke und Konzentration.

Wir haben gemeint, dafs die folgende Anordnung besondere Vorteile gewähre.

\section{Beschreibung des neuen Spektrokolorimeters.}

Die auf einem schweren Fufs befindliche Säule $S$ trägt eine Platte $P$, auf welcher die beiden Mensuren $A$ und $B$ aufgestellt werden. Dieselben besitzen eine Teilung in der Länge von $25 \mathrm{~cm}$, der Cylinder $B$ hat nur einen gewöhnlichen Hahn zum Ablassen der Flüssigkeit, der Cylinder $A$ aber aufser einem solchen, stärkeren Erniedrigungen der Oberfläche dienenden $\mathrm{Hahn}$, die seitlich angebrachte Vorrichtung $C$. durch welche das Niveau in engeren Grenzen verändert werden kann.

Über den beiden Mensuren befinden sich die beiden Reflexionsprismen $r_{1}$ und $r_{2}$, unter ihnell die beiden Prismen $r_{3}$ und $r_{4}$ und vor diesen letzten in dem Rohre $D$ ein HüFnersches Reflexionsprisma $^{1} R$. Iie Strablen der Gasflamme $G$, als welche ein gewöhnlicher Argandbrenner oder besser noch ein Auerbrenner dient. fallen, nachdem die Flamme durch den Triebknopf $T$ in die richtige Höhe gestellt worden ist, durch die Linse $L$ auf die beiden Retlexionsprismen $r_{1}$ und $r_{2}$, nach Reflexion an deren Hypotenusenflächen durch die Flüssigkeiten in den beiden Mensuren $A$ und $B$ und nach nochmaliger totaler Reflexion an den Hypotenusenflächen der Prismen $r_{3}$ und $r_{4}$ auf das Retlexionsprisma $R$; aus diesem treten sie so aus, dafs das Auge durch eine aufgesetzte Lupe $l$ zwei eng benachbarte Felder erblickt, welche nur durch die vordere feine Kante des Prismas $R$ getrennt werden.

In der bisher beschriebenen Form kann nun der Apparat als gewöhnliches Kolorimeter benutzt werden. Man überzeugt sich zunächst, beror die Mensuren $A$ und $B$ eingesetzt werden, dal's beim Hineinblicken in die Lupe $l$, mittels welcher die rordere Kante des Prismas $R$ scharf eingestellt wird, die beiden Felder gleich hell er-

1 Zeitsthr. phys. Chem. (1889) 3, 562. 
scheinen; ist solches nicht der Fall, so kann man es durch Heben oder Senken des Brenners $G$ mittels des Triebknopfes $T$ bewirken. Nun füllt man die beiden Mensuren $A$ und $B$ mit den beiden auf ihre Konzentration $\mathrm{zu}$ vergleichenden Lösungen, wobei man gut thut, die stärker konzentrierte in die Mensur $A$ zu füllen. Dann kann in der Mensur $B$ die Vergleichslösung bis zum höchsten Teilstrich 25 reichen. Man ist daran natürlich nicht gebunden, ja bei dunkleren Lösungen kann es vielleicht sogar notwendig sein, eine geringere Schichtendicke zu benutzen; man wählt dann irgend eine andere Höhe der Flüssigkeit im Cylinder $B$, etwa 10,15 oder $20 \mathrm{~cm}$.

Sodann wird die Höhe der Lösung in der Mensur $A$ durch den angebrachten seitlichen Abtlufshabn, sowie endlich durch die Vorrichtung $C$ so eingestellt, dafs die durch die Lupe $l$ betrachteten beiden Felder in gleicherHelligkeit erscheinen.

Wir leiten hier noch einmal die bekannte und viel benutzte Formel über die Beziehung zwischen Dicke der Fluissigkeitsschicht und Konzentration $a b$, einesteils der Vollständigkeit halber, und

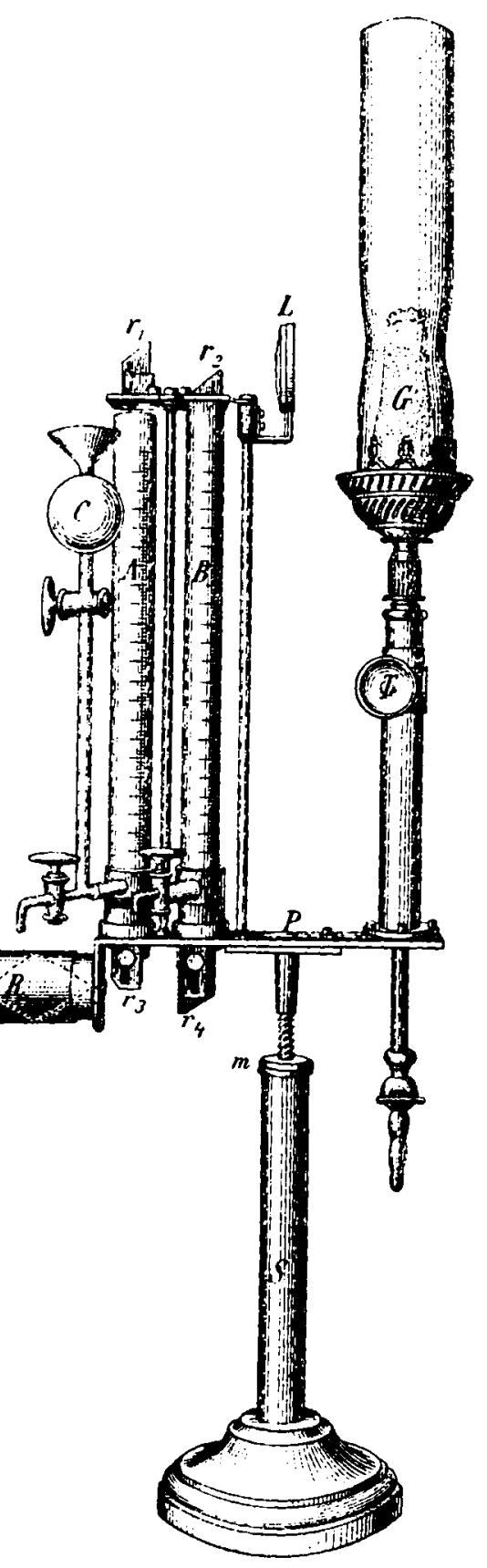


dann auch, um sie in Zusammenhang mit unseren vorstehenden Entwickelungen $\mathrm{zu}$ bringen.

Es seien die Schichtendicken in den beiden Cylindern $m_{1}$ und $m_{2}$, die zugehörigen Konzentrationen der Lösungen $c_{1}$ und $c_{2}$ und die übrigbleibenden Lichtstärken $J_{1}$ und $J_{2}$, während die Helligkeit des auf die Oberfläche beider Lösungen fallenden Lichtes $J$ ist.

Dann ist entsprechend der Gleichung (7)

$$
\begin{aligned}
& J_{1}=\stackrel{J}{n^{m_{1} c_{1}}}, \\
& J_{2}=\frac{J}{n^{n+w_{2} c_{2}}} \cdot .
\end{aligned}
$$

Sind die übrigbleibenden Lichtstärken durch entsprechende Veränderung der Schichtenhöhen einander gleich gemacht, also $J_{1}=J_{2}$, so ist

also

$$
\frac{J}{n^{m_{1} c_{1}}}=-\frac{J}{n^{m_{2}}-\overline{c_{2}}}
$$

oder

$$
m_{1} c_{1}=m_{2} c_{2}
$$

$$
m_{1}=\frac{c_{2}}{m_{1}}
$$

d. h. bei zwei verschieden konzentrierten Lösungen desselben Körpers in derselben Lösungsflüssigkeit sind Schichtendicke und Konzentration einander umgekehrt proportional unter der Voraussetzung, dafs die Schichtendicken so eingestellt sind, dafs beide Lösungen die gleiche Lichtabsorption ausüben.

Ist $e_{1}$ bekannt, so findet sich also durch Herbeiführung gleicher Helligkeit in den beiden Cylindern die unbekannte Konzentration $e_{2}$ aus der einfachen Beziehung

$$
c_{2}=-\frac{m_{1}}{m_{2}}-c_{1} .
$$

Soll der beschriebene Apparat zu quantitativ-spektralanalytischer Untersuchung benutzt werden, so wird $\operatorname{der}$ Cylinder $B$ mit der Lösungsflüssigkeit gefüllt, der Cylinder $A$ dagegen mit der zu untersuchenden Lösung.

Dann wird der kolorimetriscle Apparat vor den Spalt eines Spektrophotometers gestellt, nachdem zuvor die Lupe $l$ entfernt worden ist. Es kann dann die vordere Kante des Reflexionsprismas $R$ in unnittelbare Berïhrung mit den Spaltschneiden gebracht werden, so dafs ihr Bild, welches die Trennungslinie der beiden Spektren- 
hälften im Gesichtsfelde bildet, gleichzeitig mit dem Bilde des Spaltes scharf erscheint. Es kamn ferner durch Hebung oder Senkung des ganzen kolorimetrischen Apparates, welches durch Irehen der Mutter $m$ an der Tragsäule $s$ bewirkt wird, die andere Kante des Reflexionsprismas $R$ genau in die Mitte der Länge des Spaltes gebracht werden.

Wie bereits oben angedeutet, müssen diann die Lichtregulierungseinrichtungen des Spektrophotometers - sei es der VIERordtsche Doppelspalt oder etwaige Polarisationseinrichtungen - so eingestellt werden, dals die der oberen Spalthälfte entsprechende Spektrumhälfte im Gesichtsfelde von vornherein nur $1 / 10$ so hell ist, wie die der unteren Spalthälfte entsprechende. Man ersieht nämlich aus dem Verlauf der Strahlen, dals die durch den mit der Lösungsflüssigkeit zu füllenden Cylinder $B$ tretenden Lichtstrahlen beim Austritt aus dem Reflexionsprisma $R$ sich oberhalb der Mittelliante befinden, die clurch die Lösung selbst im Cylinder $A$ dagegen unterhalb derselben.

Hierauf ist die Höhe der Lösung im Cylinder $A$ mittels des Hahnes und der Einstellungsvorrichtung $C$ so einzustellen, dals die beiden Hälften des im Gesichtsfelde abgeblendeten Spektralbezirkes die gleiche Helligkeit haben. Es empfiehlt sich, um ganz genaue Resultate zu erzielen, nach der in dieser Weise vorläufig bewirkten Finstellung, die Höhe der Lösungsfiüssigkeit im Cylinder $B$ auf den gleichen Betrag zu bringen, wie diejenige der Lösung im Cylinder $A$, und um eine nochmalige wahrscheinlich nur sehr geringe Veränderung der Schichtendicke im Cylinder $A$ zu bewirken.

Liest man nun die Höhe der Lösung im Cylinder $A$ mit $m$ ab, so ist die Konzentration der Lösung nach Gleichung (9a)

$$
c=\frac{A}{m}
$$

anf höchst einfache und übersichtliche Weise bestimmt. ${ }^{1}$

${ }^{1}$ Durch das Ableben meines Bruders ist es nicht möglich geworden, die Brauchbarkeit des beschriebenen Apparates vor dieser Veröffentlichung durch Versuche darznthun und durch Mitteilung solcher Versuchsergebnisse zu belegen. Es mufs dieses deshalb anderen Fachlenten überlassen bleiben, welche den in Obigem mitgeteilten Gedanken ihr Interesse zuwenden wollen.

Bei der Redaktion eingegangen am 13. Juni 1895. 